\title{
CUTTING PARAMETERS AND VIBRATIONS ANALYSIS OF MAGNETIC BEARING SPINDLE IN MILLING PROCESS
}

\author{
Amel Bouaziz, Maher Barkallah, Slim Bouaziz \\ Laboratory of Mechanical Modeling and Production, National School of Engineers of Sfax, University of Sfax, Tunisia \\ e-mail: slim.bouaziz1@gmail.com \\ JeAn Yves Choley \\ Laboratory of Engineering in Mechanical Systems and Materials, SUPMECA, Saint-Ouen, France
}

MOHAMED HADDAR

Laboratory of Mechanical Modeling and Production, National School of Engineers of Sfax, University of Sfax, Tunisia

\begin{abstract}
In modern production, milling is considered the widespread cutting process in the formatting field. It remains important to study this manufacturing process as it can be subject to some parasitic phenomena that can degrade surface roughness of the machined part, increase tool wear and reduce spindle life span. In fact, the best quality work piece is obtained with a suitable choice of parameters and cutting conditions. In another hand, the study of tool vibrations and the cutting force attitude is related to the study of bearings as they present an essential part in the spindle system. In this work, a modeling of a High Speed Milling (HSM) spindle supported by two pair of Active Magnetic Bearings (AMB) is presented. The spindle is modeled by Timoshenko beam finite elements where six degrees of freedom are taken into account. The rigid displacements are also introduced in the modeling. Gyroscopic and centrifugal terms are included in the general equation. The bearings reaction forces are modeled as linear functions of journal displacement and velocity in the bearing clearance. A cutting force model for peripheral milling is proposed to estimate the tool-tip dynamic responses as well as dynamic cutting forces which are also numerically investigated. The time history of response, orbit, FFT diagram at the tool-tip center and the bearings dynamic coefficients are plotted to analyze dynamic behavior of the spindle.
\end{abstract}

Keywords: milling process, cutting forces, chip thickness, dynamic coefficients, orbit

\section{Introduction}

During the last years, High Speed Milling (HSM) process have become one of the most popular in the industry of shaping and producing mechanical parts and molds. It allows having complex forms thanks to the variety of cutting tools composed of several cutting edges and driven by rotational motion. There are multiple parameters that influence the forces acting on the cutter. Their knowledge and prediction become important in order to favor the adaptation of cutting tools conditions. The last researches were concentrated on studying the milling spindle with different types of bearings, especially AMB as they present many advantages deduced by Knospe (2007). He showed that AMB were characterized by their accuracy, high robustness to shock and high rotational speed, which was also proved by Kimman et al. (2010) and Gourc et al. (2011). Also, among the special feature of AMB, the possibility to vary the number of electromagnets is treated in literature. Indeed, Bouaziz et al. $(2011,2013)$ studied that variation effect on the dynamic behavior of a rigid rotor with a misalignment defect. They demonstrated that the vibratory level of the rotor decreased with an increase in the electromagnets number. Belhadj Messaoud et al. (2011) presented the effect of the air gap and rotor speed on electromagnetic forces. From the results obtained in that work, they concluded that the electromagnetic forces 
intensity increased when the air gap decreased, but it remained unresponsive according to the rotor speed level.

In addition, it is possible to use various components of AMB such as sensors and feedback currents to predict cutting forces. In fact, Auchet et al. (2004) expanded a new method to measure cutting forces by analyzing command voltages of AMB. This cutting force has an influence on dimensional accuracy due to the tool and work piece deflection in peripheral milling. For this, a theoretical dynamic cutting force model was presented by Liu et al. (2002) including the size effect of not deformed chip thickness, the impact of the effective rake angle and the chip flow angle. Numerical simulation and prediction of cutting forces in five-axis milling processes with cutter run-out and based on tool motion analysis was presented by Sun and Guo (2011). The predicted cutting forces illustrate good accordance with experimental results that the tool orientation angle and cutting depth vary continuously for both the specified cutting conditions and milling cases. Relative to classical methods, that proposed method permits one to predict cutting forces with a higher accuracy, and it is able to be directly used in five-axis milling. Lai (2000) studied the influence of dynamic radii, cutting feed rate, and radial and axial depths of cut on milling forces. He concluded that the chip thickness presented the most significant influence. In the same context, Klocke et al. (2009) investigated the influence of cutting parameters in micro milling on the surface quality and tool life such as cutting speed and feed per tooth. As a results, they showed that the feed per tooth and feed rate extremely affected the surface quality in micro milling. In fact, to increase the surface quality, it is necessary to decrease the feed rate value. From an analytical prediction of the cutting force, Fontaine et al. (2007) presented a method to optimize the milling tools geometry. The influence of geometrical parameters like helix angle, rake angle and tool tip envelope radius was studied. The author demonstrates the ability of that type of model to provide optimization criteria for the design and selection of cutting tools. Budak (2006a,b) presented the milling force, workpiece and tool deflection, form error and stability models. From the used method, he checked the process constraints and selected the optimal cutting conditions. Concerning the machining process stability, Faassen et al. (2003) developed a dynamic model for milling process in which the stability lobes were generated. This model structurally predicts the stability limit slightly too conservative. Another new to the dynamical modeling of AMB to identify machining stability was presented by Gourc et al. (2011). From that model, the authors concluded that the machining process stability was sensitive to the position of nodes of mode of the flexible rotor. Also, they confirmed that it was important to take in consideration strong forced vibrations as they could cause loss off safety. The stability of a high speed spindle system in the presence of the gyroscopic effect was investigated by Movahhedy and Mosaddegh (2006). In that study, it has found that the stability lobe predictions based on stationary FRFs were not conservative when the gyroscopic effects were respectable and that the gyroscopic effects became significant only at very high speeds compared with conventional speeds. Also, Gagnol et al. (2007) developed and experimentally validated an integrated spindle finite-element model in order to characterize the dynamic behavior of a motorized machine tool spindle. They demonstrated the dependence of dynamic stiffness on spindle speed. Using this model, a new stability lobes diagram was proposed. A research performed by Zatarain et al. (2006) showed that mill helix angle could play an important role in instability due to repetitive impact driven chatter. In order to predict the occurrence of chatter vibrations, Lacerda and Lima (2004) applied an analytical method in which the time-varying directional dynamic milling forces coefficients were expanded in Fourier series and integrated along width of the cut bound by entry and exit angles. Wan et al. (2010) proposed a unified method for predicting stability lobes of the milling process with multiple delays. It was found that feed per tooth had great influence on the stability lobes when cutter run out occurred.

In this paper, a HSM spindle with AMB is modeled by the finite element method based on the Timoshenko beam theory and used by Nelson and McVough (1976) and Nelson (1980). Six 
degrees of freedom are considered. Rigid displacements are also taken in account (Hentati et al., 2013). AMB are presented as spring and damper elements. Peripheral milling process is modeled and cutting forces are formulated. The dynamic response at the spindle tool-tip, cutting forces in $x$-, $y$ - and $z$-directions and the influence of some cutting parameters are predicted. The attitude of the used AMB is then investigated.

\section{Modeling of AMB spindle machining}

\subsection{Mechanical model of the spindle}

The studied spindle model is presented in Fig. 1. The modeling is based on using a new approach developed by Hentati et al. (2013). This method is based on coupling both elastic and rigid spindle deformations. So, the shaft is discretized into 23 Timoshenko beam elements with different circular sections, where six elastic degrees of freedom are taken in account. Six degrees of rigid motion are also considered. Thus, the gyroscopic effect and centrifugal force will be taken into account in this study. The unbalance is not treated. The tool holder and cutter are included as parts of the spindle system in the specifically developed finite element model.

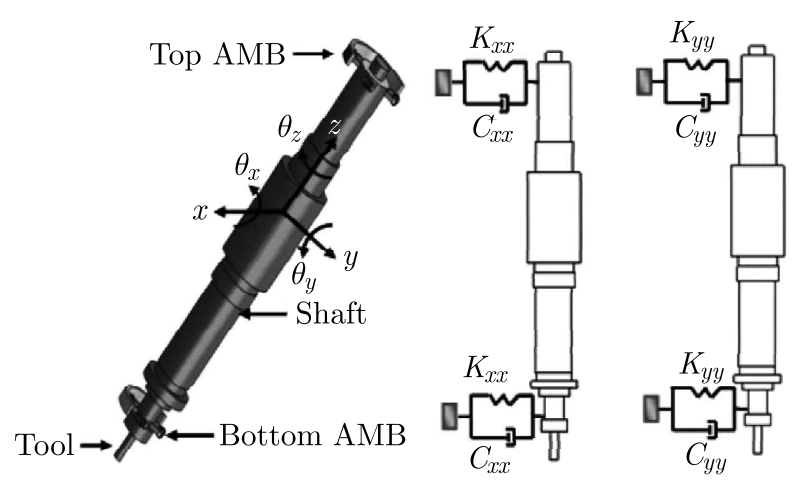

Fig. 1. Modeling of the AMB spindle machining

In this AMB spindle model we make use of a classical bearing configuration. Two radial AMBs and an axial bearing suspend the rotor in the central position. The AMB consists of four electromagnets symmetrically placed relative to the rotor. Electromagnetic forces produced by every pair of electromagnets in the $x$ - and $y$-directions are presented in the following equation (Bouaziz et al., 2011)

$$
f_{j}\left(I_{j}, u_{j}\right)=-a\left[\left(\frac{I_{0}-I_{j}}{e_{0}-u_{j}}\right)^{2}-\left(\frac{I_{0}+I_{j}}{e_{0}+u_{j}}\right)^{2}\right] \quad j=x, y
$$

where $u_{j}$ represents small deformations in the $j$-th direction and $e_{0}$ is the nominal air gap between the shaft and the stator, $a$ is the global magnetic permeability, and it is expressed as follows

$$
a=\frac{\mu_{0} S n^{2} I_{0}^{2}}{4} \cos \theta
$$

$\mu_{0}, n, S$ and $\theta$ are respectively permeability of vacuum, windings number, cross sectional area and half angle between the poles of electromagnets.

A proportional derivative controller (PD) is used to determine the control current expressed as

$$
I_{j}=k_{p} u_{j}+k_{d} \dot{u}_{j} \quad j=x, y
$$


where $\dot{u}_{j}$ is the velocity corresponding to the small deformation $u_{j}, k_{p}$ presents the proportional gain. It is assumed to be in periodic form for better stability and controllability of motion of the spindle AMB system (Bouaziz et al., 2011, 2013; Amer and Hegazy, 2007)

$$
k_{p}=k_{0}+k_{1} \cos \omega t+k_{2} \cos 2 \omega t
$$

and $k_{d}$ denotes the derivative gain.

In this model and as presented in Fig. 1, the electromagnetic field is modeled by stiffness and damping coefficients. The nonlinear electromagnetic forces at each bearing can be written in matrix form as follows (Bouaziz et al., 2011)

$$
\left[K_{i j}\right]\left\{\begin{array}{l}
u_{x} \\
u_{y}
\end{array}\right\}+\left[C_{i j}\right]\left\{\begin{array}{l}
\dot{u}_{x} \\
\dot{u}_{y}
\end{array}\right\}=\left\{\begin{array}{l}
f_{x} \\
f_{y}
\end{array}\right\}
$$

where $\left[K_{i j}\right]$ is the bearing stiffness matrix, $\left[C_{i j}\right]$ represents the bearing damping matrix

$$
\left[K_{i j}\right]=\left[\begin{array}{cc}
K_{x x} & 0 \\
0 & K_{y y}
\end{array}\right] \quad\left[C_{i j}\right]=\left[\begin{array}{cc}
C_{x x} & 0 \\
0 & C_{y y}
\end{array}\right]
$$

The axial bearing consists of two magnetic actuators on each side of the thrust element. The magnetic actuators in this setup are reluctance type actuators having a circular $u$-shaped core with a tangentially wound coil. The resulting force, $f_{z}$ of the axial bearing is linearized as

$$
f_{a b}=K_{i z} I_{z}+K_{z} u_{z}
$$

where $K_{i z}, I_{z}$ and $K_{z}$ are the force current dependencies, the control current of the actuator and the negative stiffness of the axial bearing respectively, $u_{z}$ is the axial displacement of the shaft.

\subsection{Model formulation of the cutting forces}

Figure 2 presents a cross-sectional view of the cutting force model in peripheral milling. The cutting force acting on the tool and the workpiece vary depending on chip thickness, shock of engagement, specific cutting pressure and generated vibrations. They appear only when the tool is in contact with the part (cutting region). The cutting force is characterized by a tangential component $F_{t}$ orthogonal to the specific segment of the cutting edge. This force is assumed to be proportional to the chip thickness and axial depth of the cut. The radial component $F_{r}$ is proportional to $F_{t}$ and orthogonal to both the cutting edge segment and the $z$-axis. The third component is the axial forc $F_{a}$. This one is typically much smaller than either $F_{t}$ or $F_{r}$ and does not contribute greatly to the bending moment produced on the cutter.

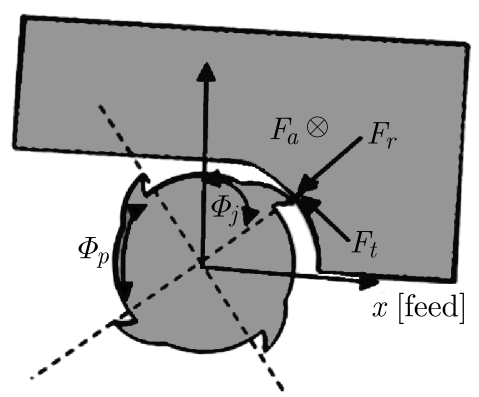

Fig. 2. Cross-sectional view of a peripheral milling process showing different forces 
The milling force variation against cutter rotation can be predicted by calculating $F_{t}, F_{r}$ and $F_{a}$ for different values of $\phi_{j}$

$$
F_{t}=K_{t} a_{p} H\left(\phi_{j}(t)\right) \quad F_{r}=K_{r} F_{t} \quad F_{a}=K_{a} F_{t}
$$

where $H\left(\phi_{j}(t)\right)$ is the instantaneous chip thickness, $\phi_{j}(t)$ is the rotation angle of tooth $j$, measured from the positive $y$-axis as shown in Fig. 2. $a_{p}, K_{t}, K_{r}$ and $K_{a}$ are axial depth and the specific coefficients of the cut.

The resulting chip thickness $H\left(\phi_{j}(t)\right)$ is composed of a static part (stationary part) $H_{s}\left(\phi_{j}(t)\right)$ due to the rigid body motion of the cutter and a dynamic component $H_{d}\left(\phi_{j}(t)\right)$ which is caused by vibrations of the tool at the present and previous tooth periods. $H_{s}\left(\phi_{j}(t)\right)$ and $H_{d}\left(\phi_{j}(t)\right)$ assume the following form

$$
\begin{aligned}
& H_{s}\left(\phi_{j}(t)\right)=f_{z} \sin \left(\phi_{j}(t)\right) \\
& H_{d}\left(\phi_{j}(t)\right)=\left[u_{x}(t)-u_{x}(t-\tau)\right] \sin \left(\phi_{j}(t)\right)-\left[u_{y}(t)-u_{y}(t-\tau)\right] \cos \left(\phi_{j}(t)\right)
\end{aligned}
$$

where $u_{x}(t)$ and $u_{y}(t)$ represent deflections of the tool-tip at the present time, $u_{x}(t-\tau)$ and $u_{y}(t-\tau)$ are deflections of the tool-tip at the previous time, $\tau$ is the tooth passing period time, defined as $\tau=60 / N Z$ and $N, Z$ are respectively the spindle speed and the teeth number of the cutter.

The rotation angle $\phi_{j}(t)$ is expressed as follows

$$
\phi_{j}(t)=\Omega t+j \Phi_{p} \quad j=0,1, \ldots, Z-1
$$

where $\Omega$ is the angular velocity in $\mathrm{rad} / \mathrm{s}$ and $\Phi_{p}$ is the angle between two subsequent teeth (pitch angle), expressed as $\Phi_{p}=2 \pi / Z$.

\subsection{Equation of motion}

As the modeling of the spindle is based on the coupling of rigid displacements and small elastic deformations, the total displacement vector is expressed as follows

$$
\mathbf{Q}=\left[U_{1}, V_{1}, W_{1}, \theta_{x_{1}}, \theta_{y_{1}}, \theta_{z_{1}}, \ldots, U_{i}, V_{i}, W_{i}, \theta_{x i}, \theta_{y_{i}}, \theta_{z_{i}}, X_{A}, Y_{A}, Z_{A}, \alpha_{x}, \alpha_{y}, \alpha_{z}\right]^{\mathrm{T}}
$$

$i$ is the number of nodes, $\left(U_{1}, V_{1}, W_{1}, \theta_{x_{1}}, \theta_{y_{1}}, \theta_{z_{1}}, \ldots, U_{i}, V_{i}, W_{i}, \theta_{x i}, \theta_{y_{i}}, \theta_{z_{i}}\right)$ presents nodal displacements, $\left(X_{A}, Y_{A}, Z_{A}, \alpha_{x}, \alpha_{y}, \alpha_{z}\right)$ are the displacements of rigid motion.

Applying the Lagrange formalism for kinetic and potential energies, the global equation of motion is

$$
\mathbf{M} \ddot{\mathbf{Q}}+\left(\mathbf{G}+\mathbf{D}+\mathbf{C}_{b}(t)\right) \dot{\mathbf{Q}}+\left(\mathbf{K}+\mathbf{K}_{b}(t)\right) \mathbf{Q}=\mathbf{F}_{c(x, y, z)}(t, \mathbf{Q})+\mathbf{f}_{a b}(t, \mathbf{Q}, \dot{\mathbf{Q}})
$$

where $\mathbf{M}$ is the global mass matrix, $\mathbf{G}$ - the global gyroscopic matrix

$$
\mathbf{M}=\left[\begin{array}{cc}
\mathbf{M}_{F} & \mathbf{M}_{R F} \\
\mathbf{M}_{R F}^{\mathrm{T}} & \mathbf{M}_{R}
\end{array}\right] \quad \mathbf{G}=2 \Omega\left[\begin{array}{cc}
\mathbf{G}_{F} & \mathbf{G}_{R F} \\
-\mathbf{G}_{R F}^{\mathrm{T}} & \mathbf{G}_{R}
\end{array}\right]
$$

$F$ and $R$ subscripts respectively represent the flexible or rigid part. $\mathbf{D}=\alpha \mathbf{M}+\beta \mathbf{K}$ presents the damping matrix numerically constructed as a linear combination of the mass and stiffness matrix, where $\alpha$ and $\beta$ are the damping coefficients. $\mathbf{C}_{b}(t)$ is the variable matrix containing the damping coefficients of bearings

$$
\mathbf{C}_{b}(t)=\left[\begin{array}{cccccccc}
0 & \cdot & \cdot & 0 & \cdot & \cdot & 0 & \\
& \cdot & \cdot & \cdot & \cdot & \cdot & \cdot & 0 \\
\cdot & C_{x x} & 0 & \cdot & \cdot & \cdot & \cdot & \\
0 & 0 & C_{y y} & \cdot & \cdot & \cdot & \cdot \\
\cdot & \cdot & \cdot & 0 & C_{x x} & 0 & \cdot \\
\cdot & \cdot & \cdot & \cdot & C_{y y} & 0 & \\
0 & \cdot & \cdot & \cdot & 0 & \cdot & 0
\end{array}\right]
$$


$\mathbf{K}$ is the global stiffness matrix, $\mathbf{K}_{c}$ - the global centrifugal matrix

$$
\mathbf{K}=\left[\begin{array}{cc}
\mathbf{K}_{F} & \mathbf{0} \\
\mathbf{0} & \mathbf{0}
\end{array}\right]-\underbrace{\Omega^{2}\left[\begin{array}{cc}
\mathbf{C}_{F} & \mathbf{0} \\
\mathbf{0} & \mathbf{0}
\end{array}\right]}_{\mathbf{K}_{c}}
$$

$\mathbf{K}_{b}(t)$ is the variable matrix containing the stiffness coefficients of bearings

$$
\mathbf{K}_{b}(t)=\left[\begin{array}{ccccccc}
0 & \cdot & \cdot & 0 & \cdot & \cdot & 0 \\
\cdot & \cdot & \cdot & \cdot & \cdot & \cdot & 0 \\
\cdot & K_{x x} & 0 & \cdot & \cdot & \cdot & \cdot \\
0 & 0 & K_{y y} & \cdot & \cdot & \cdot & \cdot \\
\cdot & \cdot & \cdot & 0 & K_{x x} & 0 & \cdot \\
\cdot & \cdot & \cdot & \cdot & 0 & K_{y y} & 0 \\
0 & \cdot & \cdot & \cdot & 0 & \cdot & 0
\end{array}\right]
$$

and $\mathbf{F}_{c(x, y, z)}(t, \mathbf{Q})$ denotes the cutting forces in the $x$-, $y$ - and $z$-directions, respectively, $\mathbf{f}_{a b}(t, \mathbf{Q}, \dot{\mathbf{Q}})$ is the electromagnetic force vector exerted by the axial bearing.

\section{Results and discussions}

In this Section, simulations are based on the spindle system with parameters listed in Tables 1 and 2. The general dynamic equation is solved by the method of resolution by Newmark coupled with Newton Raphson.

Table 1. Spindle parameters

\begin{tabular}{|l|c|c|c|}
\hline \multicolumn{1}{|c|}{ Parameter } & Symbol & Value & Unit \\
\hline \hline Permeability of vacuum & $\mu_{0}$ & $4 \pi \cdot 10^{-7}$ & $\mathrm{~Wb} / \mathrm{Am}$ \\
\hline Air gap between stator and shaft & $e_{0}$ & 0.8 & $\mathrm{~mm}$ \\
\hline Effective cross-sectional area of one electromagnet & $S$ & 200 & $\mathrm{~mm}^{2}$ \\
\hline Number of windings around the core & $n$ & 300 & - \\
\hline Half angle between poles of electromagnet & $\theta$ & 22.5 & $\mathrm{deg}$ \\
\hline Bias current & $I_{0}$ & 3 & $\mathrm{~A}$ \\
\hline Rotor angular velocity & $N$ & 20000 & $\mathrm{rpm}$ \\
\hline Rotor length & $L$ & 651.95 & $\mathrm{~mm}$ \\
\hline Stiffness coefficients & $K_{x x}, K_{x y}, K_{y x}, K_{y y}$ & - & $\mathrm{N} / \mathrm{m}$ \\
\hline Damping coefficients & $C_{x x}, C_{x y}, C_{y x}, C_{y y}$ & - & $\mathrm{Ns} / \mathrm{m}$ \\
\hline Derivative gain & $k_{d}$ & 42.4 & $\mathrm{As} / \mathrm{m}$ \\
\hline Proportional gain constant & $k_{0}$ & 4520 & - \\
\cline { 2 - 4 } & $k_{1}$ & 14869 & - \\
\cline { 2 - 5 } & $k_{2}$ & 14869 & - \\
\hline Young's modulus & $E$ & $2.1 \cdot 10^{11}$ & $\mathrm{~Pa}$ \\
\hline Density & $\rho$ & 7.85 & $\mathrm{~g} / \mathrm{cm}^{3}$ \\
\hline Poisson's ratio & $\nu$ & 0.3 & - \\
\hline Moment of inertia & $I$ & 0.136 & $\mathrm{kgm}{ }^{2}$ \\
\hline
\end{tabular}

The time responses of the tool tip are plotted in Fig. 3. From Fig. 3a, some transient effects can be observed during the first cycle while a steady state is achieved after that. Their magnitude is close. In fact, before the cutter is fully engaged, the arc of engagement increases gradually while 
Table 2. Cutting parameters

\begin{tabular}{|l|c|c|c|}
\hline \multicolumn{1}{|c|}{ Parameter } & Symbol & Value & Unit \\
\hline \hline Feed per tooth & $f_{z}$ & 0.16 & $\mathrm{~mm}$ \\
\hline Axial depth of cut & $a_{p}$ & 5 & $\mathrm{~mm}$ \\
\hline Tangential cutting coefficient & $K_{t}$ & 644 & $\mathrm{~N} / \mathrm{mm}^{2}$ \\
\hline Radial cutting coefficient & $K_{r}$ & 0.38 & $\mathrm{~N} / \mathrm{mm}^{2}$ \\
\hline Axial cutting coefficient & $K_{a}$ & 0.25 & $\mathrm{~N} / \mathrm{mm}^{2}$ \\
\hline Teeth number & $Z$ & 2 & - \\
\hline
\end{tabular}

(a)

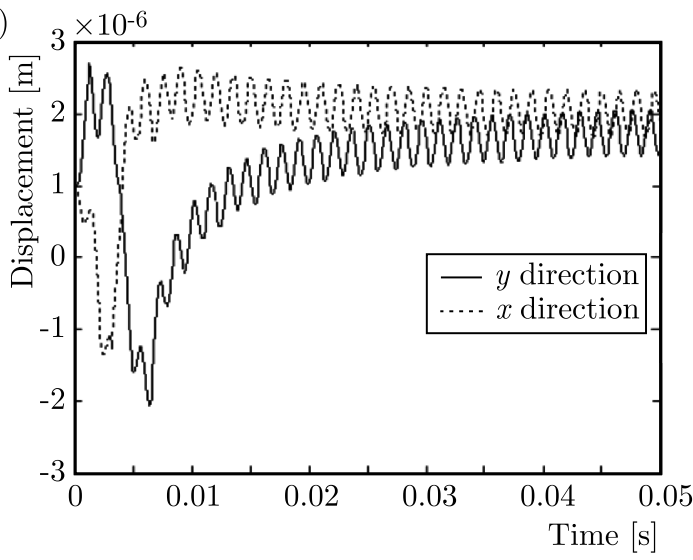

(b)

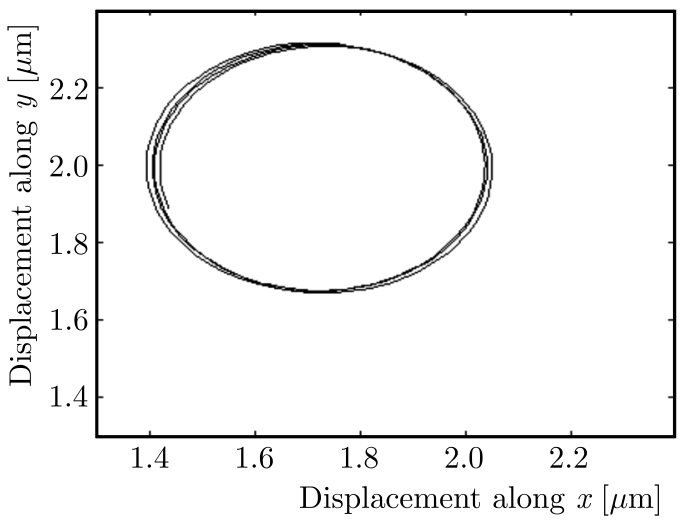

Fig. 3. The time response of the tool tip: (a) displacement of the tool tip, (b) orbit of the tool tip

the cutter is entering progressively in the cutting zone. Consequently, this leads to a gradual increase of vibrations.

The orbit of the tool tip has an elliptical shape explained by the introduction of flexible bearings (stiffness coefficient) that make the system asymmetric. Therefore, we note that vibrations in the $x$ - and $y$-directions are different from the elliptic trajectory.

The Fast Fourier Transformation (FFT) diagram for the $x$-response of the tool-tip with two teeth at a spindle speed of $20000 \mathrm{rpm}$ is shown in Fig. 4. It is found that two frequencies govern the behavior of the tool-tip response. The major peak corresponds to the frequency of $2 F_{r}(666.66 \mathrm{~Hz})$ which occurs with the cutting force frequency. An obvious low frequency peak, corresponding with the rotation frequency, is also found.

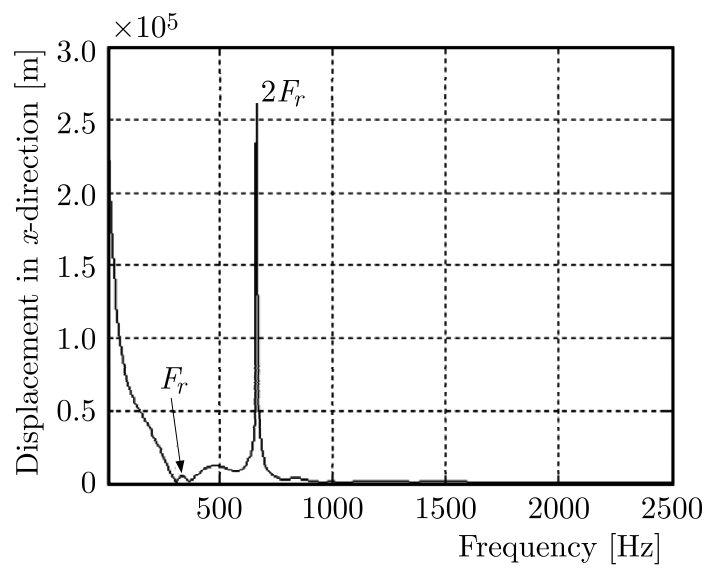

Fig. 4. Response of the tool-tip in the $x$-direction with 2 teeth 
The dynamic cutting force in the $x$-, $y$ - and $z$-directions for a two teeth cutter are presented in Fig. 5. It can be seen that the cutting force is constant as the cutter is always in contact with the matter and where the evolution of radial engagement is continuous. All the cutting components have periodic and sinusoidal behavior with a period of time equal to the half of the rotation period $\left(0.5 T_{r}\right)$.

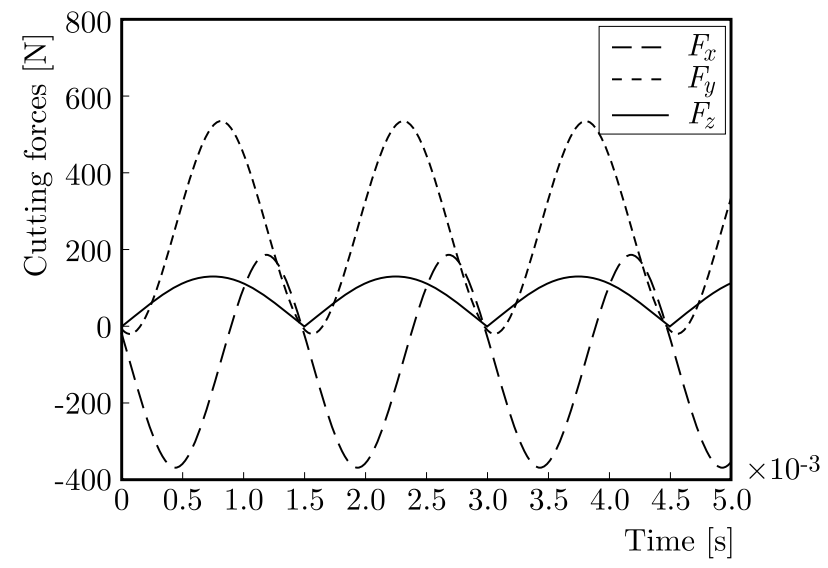

Fig. 5. Cutting forces in the $x$-, $y$ - and $z$-directions as function of time

The following part of the study is devoted to presentation of the impact of some parameters involved in the cutting forces such as the teeth number, feed per tooth and speed rotation. Figure 6 presents variation of the cutting forces for different values of feed per tooth: $0.1,0.2$ and $0.3 \mathrm{~mm}$. It appears that the components in the $x$ - and $y$-directions increase with an increase in the feed. It is worth noticing that when $f_{z}=0.1 \mathrm{~mm}$, the maximum value of $F_{y}$ is greater than the half value as $f_{z}=0.3 \mathrm{~mm}$. This evolution is logic and is explained by the fact that the feed is involved in the cut section. In fact, if the feed increases, the cutting section also increases and, therefore, the cutting force increases. This result was found by Liu et al. (2002) who also revealed that this variation was relative to the size effect of the chip thickness. In addition, we note that the $F_{y}$ component rises with a greater rate.

(a)

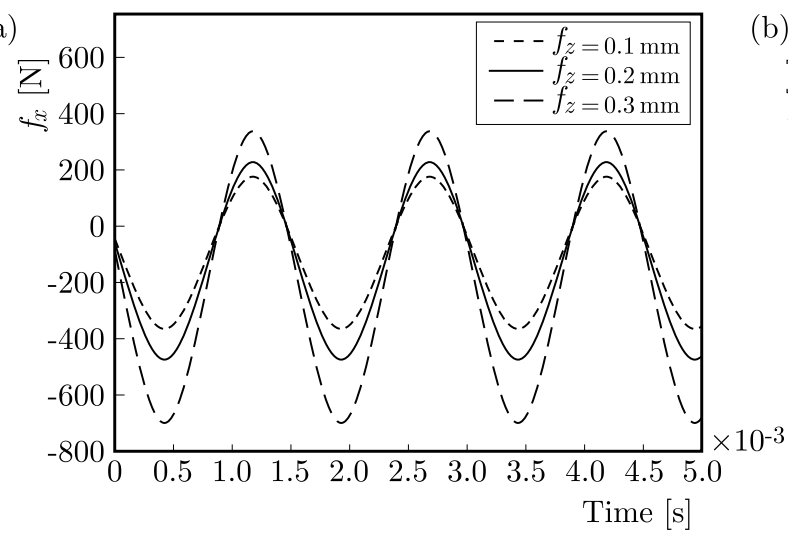

(b)

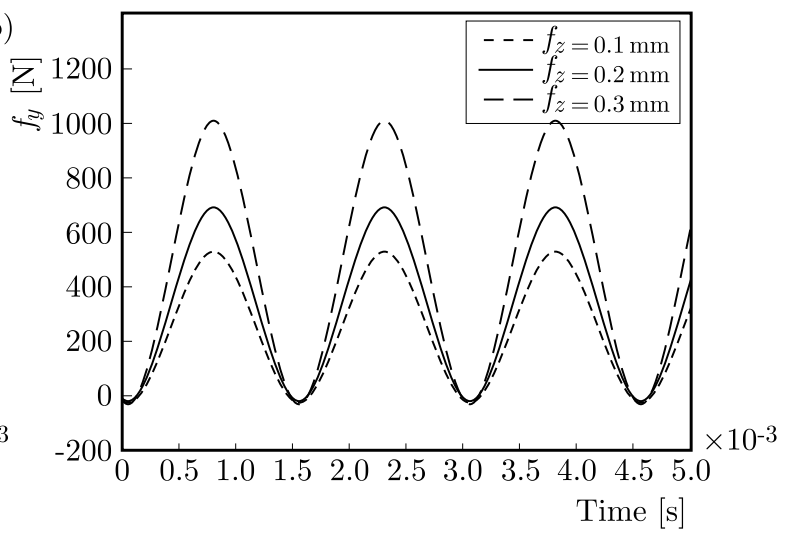

Fig. 6. Predicted cutting forces for different feed per tooth: (a) $x$-direction, (b) $y$-direction

Figure 7 shows axial depth of the cut effects the cutting force value. When this parameter changes, the cutting force vary significantly. It increases when the cutting depth increases. This raise is explained by the increase of the width of chip. For these values of axial cutting depth, we find that the cutting forces $F_{y}$ are always more important than the cutting force $F_{x}$.

Figure 8a shows the instantaneous cutting forces for the tooth number $Z=3$. It can be seen from variation of $F_{y}$ that the cutting operation starts when the second tooth comes out, with the maximum value reaching $580 \mathrm{~N}$. 

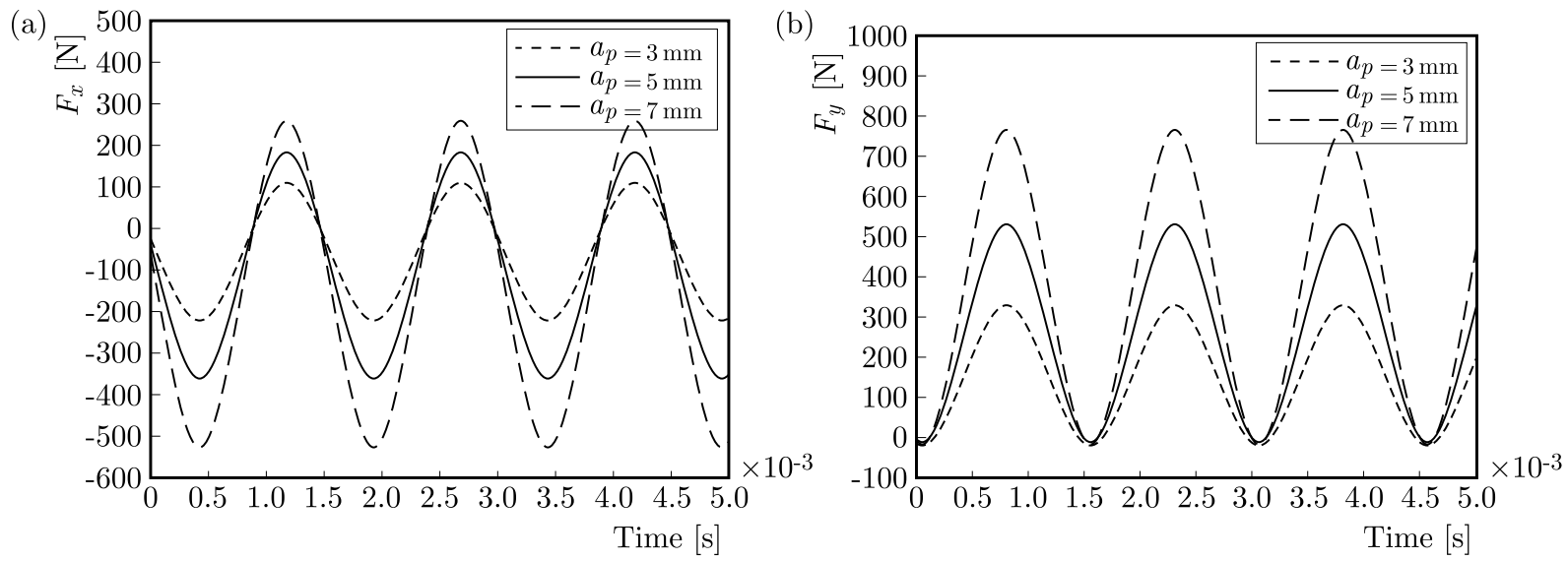

Fig. 7. Predicted cutting forces for various cutting depth: (a) $x$-direction, (b) $y$-direction

(a)

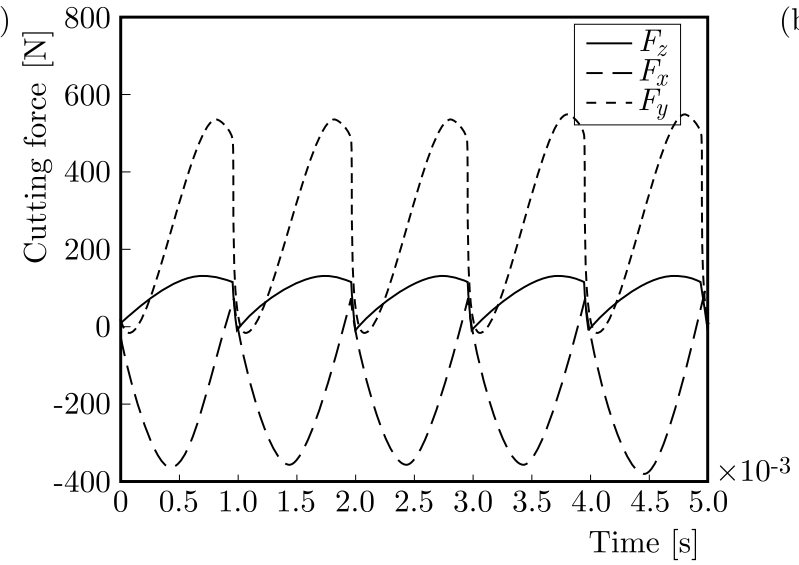

(b)

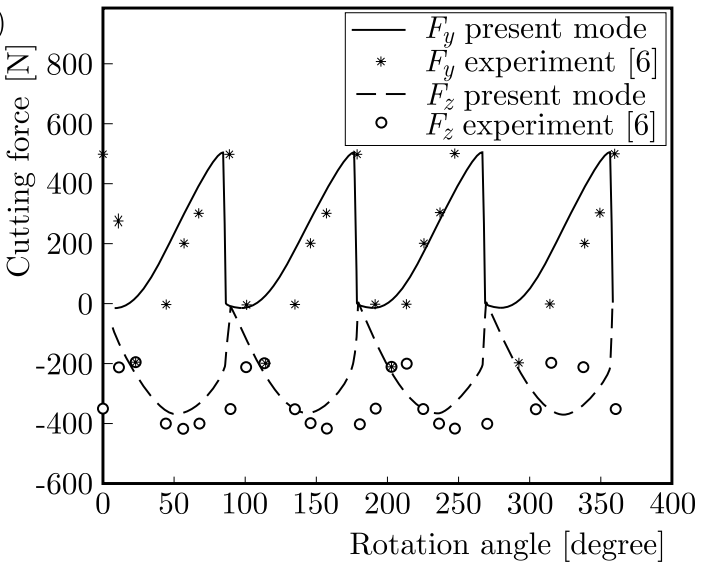

Fig. 8. Cutting force in the $x$-, $y$ - and $z$-directions: (a) $Z=3$ teeth, (b) $Z=4$ teeth

Although the shape of cutting forces is affected and changed compared to that with two teeth, the distribution is still continuous. This difference is explained by the summation of cutting forces of teeth in attack with the mutter. So, it is possible that the number of teeth variation will significantly influence the accuracy of the finished part. Figure $8 \mathrm{~b}$ also presents the predicted values of the cutting force for the same conditions but for a four teeth tool. It is noticed that the shape and values for the cutting force components $F_{x}$ and $F_{y}$ are similar to those of the cutter with three teeth. Also, these levels for the cutting forces, for a four flutes, seems to be typical for peripheral milling as this result is approximately close to the experimental ones found by Budak (2006a). The percentage ratio of the difference between the absolute maximum predicted and measured value relative to the measured value represents an error of $9 \%$.

The variation of dynamic coefficients $\left(K_{x x}, K_{y y}\right)$ is presented in Fig. 9. It is clear that the curves have a periodic form with amplitude reaching $1.8 \cdot 10^{7} \mathrm{~N} / \mathrm{m}$. The coefficient $K_{x x}$ is less important than $K_{y y}$, that is why we have obtained in Fig. 3 vibrations in the $x$-direction more severe than in the $y$-direction.

The damping coefficients, presented in Fig. 10, have low amplitude varying from about $-4.1535 \cdot 10^{4}$ to $-4.15345 \cdot 10^{4} \mathrm{Ns} / \mathrm{m}$ with instability in the beginning.

Figure 11 shows the effect of the nominal air gap on the dynamic coefficients. This result reveals that the amplitude of both stiffness and damping coefficients decrease with an increase in the nominal air gap. This result was also proved by Bouaziz et al. (2011).

The impact of the bias current I0 on the dynamic coefficients is presented in Fig. 12. For different values of $I_{0}: I_{0}=5 \mathrm{~A}, I_{0}=6 \mathrm{~A}$ and $I_{0}=6.5 \mathrm{~A}$, it is found that $K_{x x}$ and $C_{x x}$ rise when 

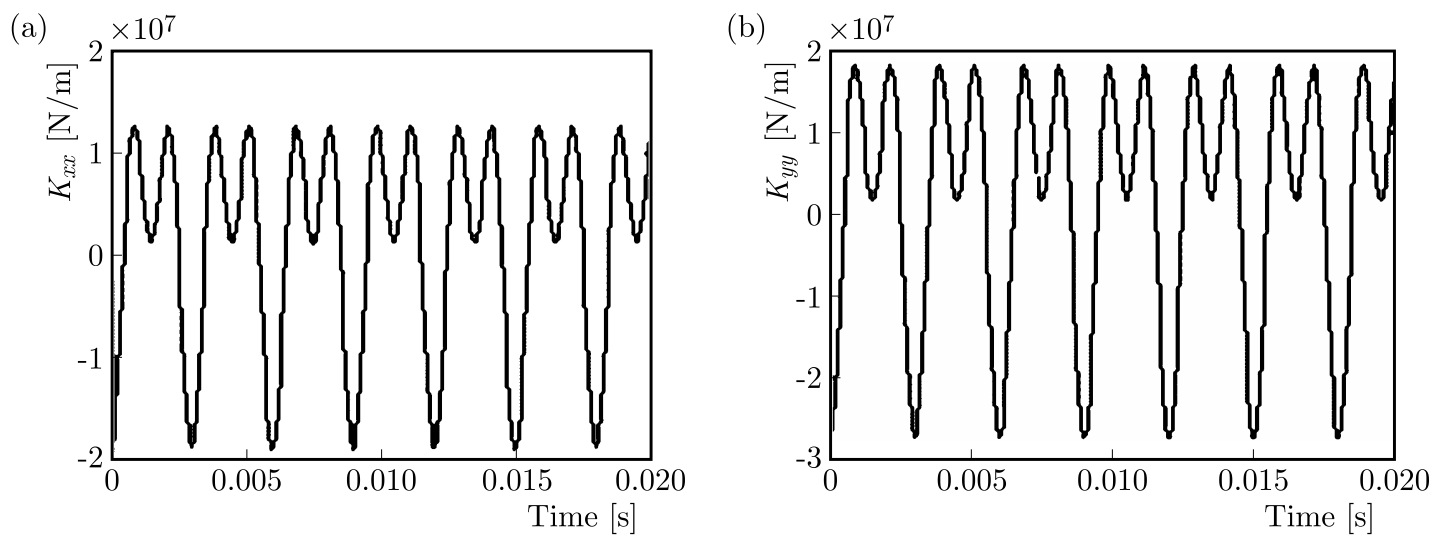

Fig. 9. Stiffness coefficients of the bottom AMB in the $x$ - and $y$-directions
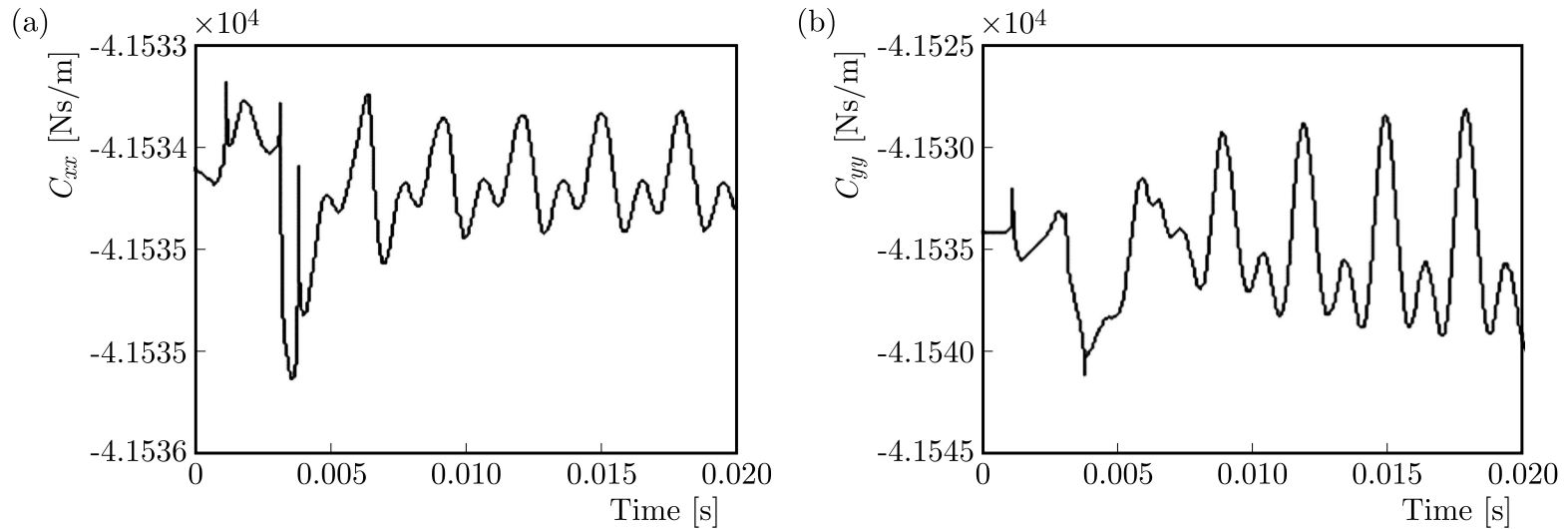

Fig. 10. Damping coefficients of the bottom AMB in the $x$ - and $y$-directions
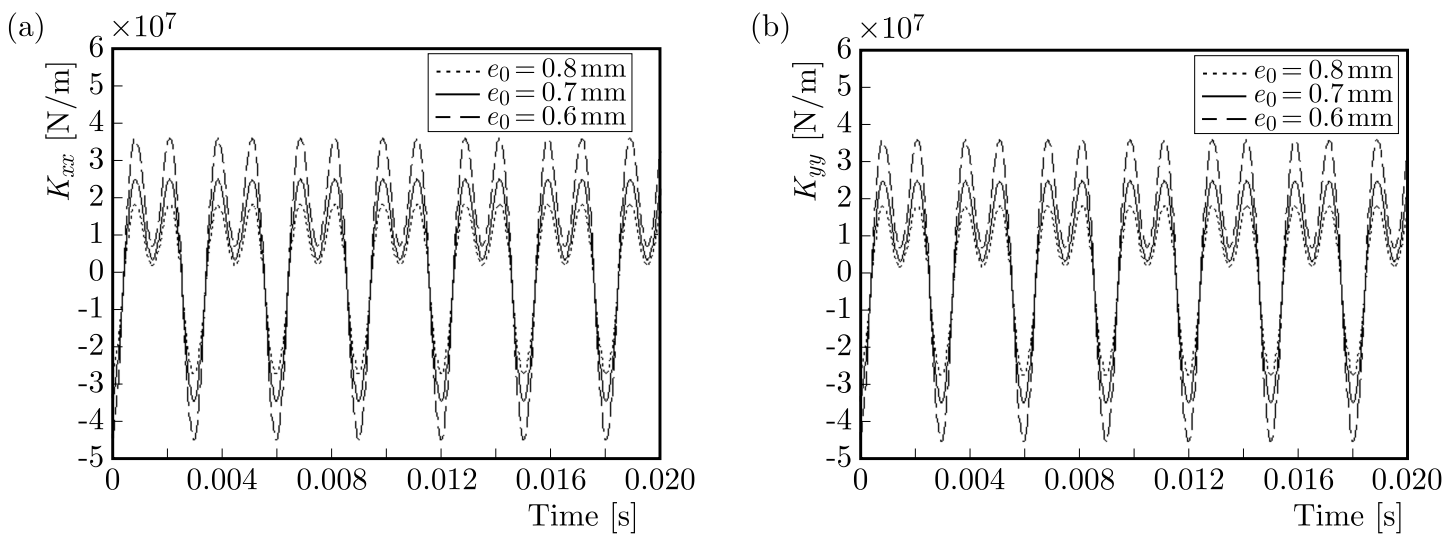

Fig. 11. Dynamic coefficients variation at the bottom AMB in the $x$-direction as function of $e_{0}$

$I_{0}$ increases. Indeed, equation (2.1) shows that the electromagnetic forces are proportional to the bias current. Therefore, the damping and stiffness coefficients should be increased to minimize the fluctuations.

Figure 13 presents variation of the stiffness and damping coefficients for rotational speeds of $N=20000 \mathrm{rpm}$ and $N=40000 \mathrm{rpm}$, respectively. We remark that the damping coefficients increase in a noticeable way relative to the stiffness coefficients. This is explained by the fact that the vibrations generated with the highest rotational speed should be absorbed and reduced due to damping. A too small change is noted for the stiffness coefficients at the beginning. 
(a)

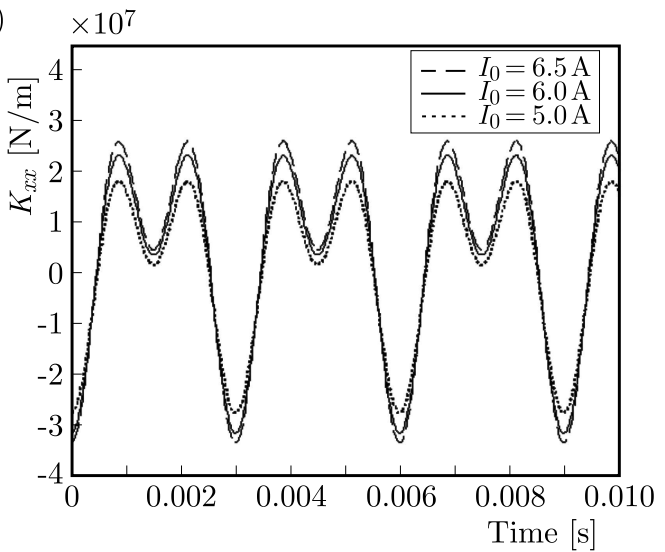

(b)

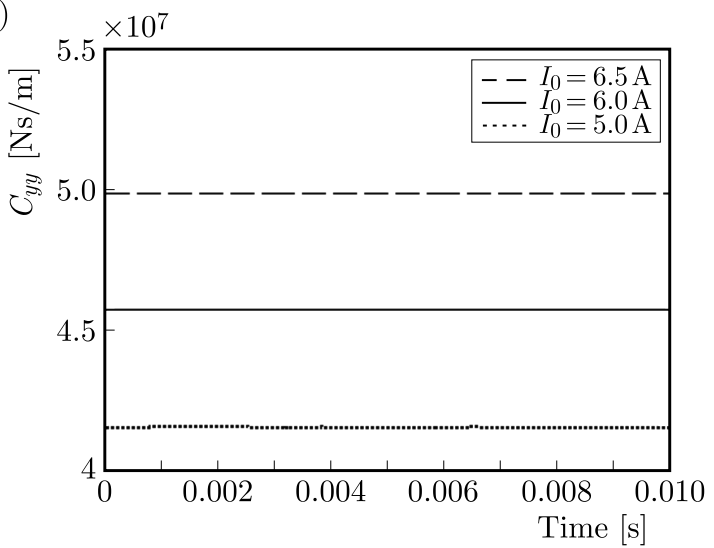

Fig. 12. Dynamic coefficients variation at the bottom AMB in the $x$-direction as function of $I_{0}$

(a)

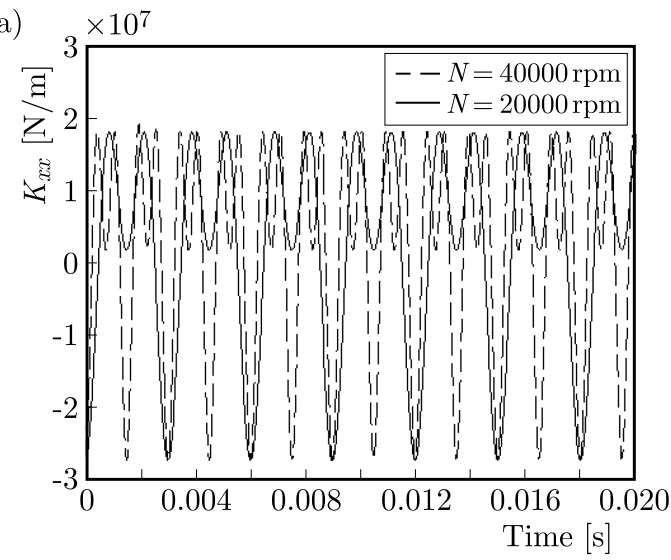

(b)

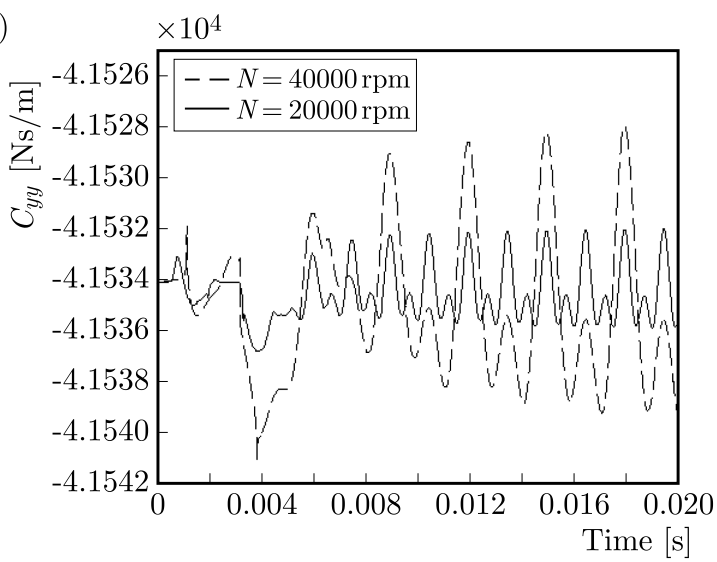

Fig. 13. Dynamic coefficients of the bottom AMB in the $x$-direction as function of rotation speed

\section{Conclusion}

This study presents dynamical analysis of a high speed AMB spindle in the peripheral milling process. The spindle rotor is modeled by finite elements using the Timoshenko beam theory. The rigid motions are also considered. A mechanistic model of the peripheral milling is presented including the influence of instantaneous chip thickness. To solve the general equations of motion, the Newmark coupled with the Newton Raphson numerical method is used. The solution gives the spindle dynamic response and explains variation of the cutting force. Analyzing this work, we conclude that the cutting force is related to the cutting parameters introduced in the modeling such as the effect of thickness of the formed chip, feed per tooth, cutting depth and the number of teeth in the cutter. So, it is necessary to select a suitable cutter with the determined flute number in order to obtain an ideal cutting force distribution. This ideality appears first, when the absolute value of the cutting force perpendicular to the feed direction during the cutting process is as small as possible; secondly, when the cutting force distribution is continuous. Concerning the modeling of the bearing, it is clear that the dynamic coefficients are influenced by the air gap as they increase when this parameter decreases. Also they increase with an increase in the rotational speed. 


\section{References}

1. Amer Y.A., Hegazy U.H., 2007, Resonance behavior of a rotor-active magnetic bearing with time-varying stiffness, Chaos, Solitons and Fractals, 34, 1328-1345

2. Auchet S., Chevrier P., Laccour M., Lipinski P., 2004, A new method of cutting force measurement based on command voltages of active Electro-Magnetic bearing, International Journal of Machine Tool and Manufacture, 44, 1441-1449

3. Belhadj Messaoud N., Bouaziz S., Maatar M., Fakhfakh T., Haddar M., 2011, Dynamic behavior of active magnetic bearing in presence of angular misalignment defect, International Journal of Applied Mechanics, 3, 1-15

4. Bouaziz S., Belhadj Messaoud N., Choley J.Y., Mataar M., Haddar M., 2013, Transient response of a rotor-AMBs system connected by a flexible mechanical coupling, Mechatronics, 23,) $573-580$

5. Bouaziz S., Belhadj Messaoud N., Mataar M., Fakhfakh T., Haddar M., 2011, A theoretical model for analyzing the dynamic behaviour of spatial misaligned rotor with active magnetic bearings, Mechatronics, 21, 899-907

6. BuDAK E., 2006a, Analytical models for high performance milling. Part I: Cutting forces, structural deformations and tolerance integrity, International Journal of Machine Tools and Manufacture, 46, $1478-1488$

7. Budak E., 2006b, Analytical models for high performance milling. Part II: Process, dynamics and stability, International Journal of Machine Tools and Manufacture, 46, 1478-1488

8. FaAssen R.P.H., van de Wouw N., Oosterling J.A.J., Nijmeijer H., Prediction of regenerative chatter by modelling and analysis of high-speed milling, International Journal of Machine Tools and Manufacture, 43, 1437-1446

9. Flocke F., Qwito F., Arntz K., 2009, A Study of the influence of cutting parameters on micro milling of steel with cubic boron nitride (CBN) tools, Micro Machining and Micro Fabrication Process Technology XIV, 7204

10. Fontaine M., Devillez A., Dudzinski D., 2007, Optimisation de la Gómtrie d'outil en fraisage à partir de la prédiction analytique des efforts de coupe, 18éme Congrès Français de Mécanique Grenoble, 27-31

11. Gagnola V., Bouzgarroua B.C., Raya P., Barrab C., Model-based chatter stability prediction for high-speed spindles, International Journal of Machine Tools and Manufacture, 47, 11761186

12. Gourc E., Seguy S., Arnaud L., 2011, Chatter milling modeling of active magnetic bearing spindle in high-speed domain, International Journal of Machine Tools and Manufacture, 51, 928936

13. Hentati T., Bouaziz A., Bouaziz S., Cholley J.Y., Haddar M., 2013, Dynamic behavior of active magnetic bearing spindle in high speed domain, International Journal of Mechatronis and Manufacturing Systems, 6, 474-492

14. Kimman M.H., Langen H.H., Munning Schmidt R.H., 2010, A miniature milling spindle with active magnetic bearings, Mechatronics, 20, 224-235

15. Knospe C.R., 2007, Active magnetic bearing for machining application, Control Engineering Practice, 15, 307-313

16. LACERdA H.B., Lima V.T., 2004, Evaluation of cutting forces and prediction of chatter vibrations in milling, Journal of the Brazilian Society of Mechanical Sciences and Engineering, 26, 74-81

17. LAi W.H., 2000, Modeling of cutting forces in end milling operations, Tamkang Journal of Science and Engineering, 3, 15-22 
18. Liu X.W., Cheng K., Webb D., Luo X.C., 2002, Prediction of cutting force distribution and its influence on dimensional accuracy in peripheral milling, International Journal of Machine Tools and Manufacture, 42, 791-800

19. Movahhedy M., Mosaddegh P,, 2006, Prediction of chatter in high speed milling including gyroscopic effects, International Journal of Machine Tools and Manufacture, 46, 996-1001

20. Nelson H.D., 1980, A finite rotating shaft element using Timoshenko beam theory, Transactions on ASME Journal of Engineering for Industry, 102, 793-803

21. Nelson H,D., MCVough J.M., 1976, The dynamic of rotor bearing system using finite element, Transactions on ASME Journal of Engineering for Industry, 98, 593-600

22. Sun Y., Guo Q., 2011, Numerical simulation and prediction of cutting forces in five-axis milling processes with cutter run-out, International Journal of Machine Tools and Manufacture, 51, 806815

23. WAn M., Zangh W.H., DAng J.W., YAng Y., 2010, A unified stability prediction method for milling process with multiple delays, International Journal of Machine Tools and Manufacture, 50, 29-41

24. Zatarain M., Muñoa J., Peigné G., Insperger T., 2006, Analysis of the influence of mill helix angle on chatter stability, CIRP Annals - Manufacturing Technology, 55, 365-368

Manuscript received May 1, 2015; accepted for print October 9, 2015 